\title{
EDIFICATE
}

I Congreso de Escuelas de Edificación y Arquitectura Técnica de España

València, 4 y 5 de noviembre de 2021

Escuela Técnica Superior de Ingeniería de Edificación

Universitat Politècnica de València

Doi: https://doi.org/10.4995/EDIFICATE2021.2021.13374

\section{RIARTE. EI repositorio científico y profesional de la Arquitectura e Ingeniería. Contenidos y avances}

\section{RIARTE. The scientific and professional repository of Architecture and Engineering. Contents and advances}

\author{
Joaquín Manuel Durán Álvarez ${ }^{\mathrm{a}}$, Juan López-Asiain Martínez ${ }^{\mathrm{b}}$, Alejandro Payán de \\ Tejada Alonsoc

\begin{abstract}
aUniversidad de Granada. jdaeuat@ugr.es, '•Universidad Politécnica de Madrid. apayan@arquitectura-tecnica.com
\end{abstract} \\ juan.lopezasiain@upm.es, ${ }^{\circ}$ Consejo General de la Arquitectura Técnica de España.
}

\begin{abstract}
Just 3 years ago, the Research Repository of Technical Architecture in Spain (RIARTE) was launched, promoted by the CGATE, with the idea of identifying, recording, visualising and making use of the scientific-technical production of Technical Architecture.
\end{abstract}

At present, almost 2,000 documents, including doctoral theses, scientific production documents, collegiate articles and technical reports, make up a very important documentary corpus of the profession. It has emerged as something completely new in Spain and has established itself as a first-rate tool for both the dissemination of research and professional work in TA and for the recognition of authors.

The study of the typology of the documents, the journals where they are published, the most prolific authors, the areas of importance and the more than 3,100 free descriptors, is a global overview of the research and work of Technical Architects and its transmission to the construction sector and society in general.

For this reason, a transversal and longitudinal analysis will be carried out on the areas of work and the most used journals.

Keywords: Scientific production, Technical Architecture, Building Engineering, Research areas, Spain, RIARTE, professional journal, keywords, institutional repository. 


\section{Resumen}

Apenas hace 3 años, se inició el Repositorio de Investigación de la Arquitectura Técnica de España (RIARTE), promovido por el CGATE, con la idea de identificar, registrar, visualizar y dar uso a la producción científico-técnica de la Arquitectura Técnica.

En la actualidad, casi 2.000 documentos entre tesis doctorales, documentos de producción científica, artículos e informes técnicos, configuran un Corpus documental de la profesión muy importante. Ha surgido como algo completamente nuevo en España y se ha establecido como una herramienta de primer orden para, tanto la difusión de la investigación y el trabajo profesional de la AT, como para el reconocimiento de los autores.

El estudio de la tipología de los documentos, las revistas donde se publica, los autores más prolíficos, las áreas de importancia y los más de 3.100 descriptores libres, es un recorrido global sobre la investigación y el trabajo de los Arquitectos Técnicos y su transmisión al sector de la construcción y a la sociedad en general.

Por ello se realizará un análisis transversal y longitudinal sobre las áreas de trabajo y las revistas más usadas.

Palabras clave: se incluirá una lista corta, como máximo diez palabras clave. Por ejemplo: plan de estudios, metodologías docentes, competencias, formación online, recursos tecnológicos, evaluación, prácticas de empresa, Proyecto Final de Grado, etc. 


\section{Introducción}

El repositorio RIARTE ${ }^{1}$ surgió como idea por parte del CGATE$^{2}$ en el año 2017, para estar plenamente operativo en el año 2018. En la actualidad, y después de tres años de funcionamiento y con casi 2.000 documentos técnico-científicos se configura como una herramienta de primer orden para la búsqueda de información en el sector de la construcción.

Con más de 20.000 visualizaciones en el año 2019, se está convirtiendo en una base de datos de referencia para cualquier agente de la construcción (técnicos de proyecto y ejecución de obras, especialistas en mantenimiento, en eficiencia energética, en instalaciones o materiales, constructores, investigadores, usuarios, etc.) y, además, recopila el corpus de investigación y técnico de la Arquitectura Técnica $\left(\mathrm{AT}^{3}\right)$.

Los repositorios, surgen como herramienta de la filosofía Open Access. Ésta surgió como "un nuevo modelo de difusión de la investigación científica" (Björk, Laakso, Welling, y Paeatau, 2014, p.237). El Movimiento Open Access emerge así, con fuerza, en el mundo de la publicación científica.

Como dice la Guía para la evaluación de repositorios institucionales de investigación (Azorín, et al, 2021, p. 4):

El acceso abierto (Open Access) consiste en proveer de acceso on-line a toda la información científica disponible (artículos, monografías, datos de investigación...) de forma gratuita para el lector y bajo licencia para que pueda ser usada y explotada por los investigadores, las empresas y los ciudadanos. La idea que subyace bajo el acceso abierto es que los resultados y los datos utilizados en investigaciones financiadas públicamente deberían estar disponibles para todos y tener licencias que permitan su reutilización.

La investigación se hace más efectiva y sus resultados más visibles cuando se eliminan las barreras legales, comerciales y tecnológicas de acceso a la información científica. Además, el acceso abierto previene la duplicación de esfuerzos en investigación, fomenta la transferencia tecnológica y de conocimiento y promueve la innovación. Todos los actores implicados en el sistema de comunicación de la información científica se benefician del acceso abierto a la investigación y a los datos generados por ésta.

Además (Ferreras y Merlo, 2015, p. 98) comentan que:

\footnotetext{
${ }^{1}$ Repositorio de Investigación en Arquitectura Técnica

${ }^{2}$ Consejo General de la Arquitectura Técnica de España

3 AT.- Arquitectura Técnica; Aparejadores; Grado en Ingeniería de Edificación; Grado en Edificación; Grado en Arquitectura Técnica; Grado en Ciencia y Tecnología de la Edificación; Grado en Arquitectura Técnica y Edificación
} 
el acceso abierto es una forma de amortizar la inversión en investigación de un país, al poner a disposición de los usuarios, la documentación derivada de la misma, además de ayudar a la inclusión digital de los ciudadanos al ofrecer los contenidos a texto completo a los investigadores, los docentes, los estudiantes, las instituciones y a toda la ciudadanía.

Desde la Declaración de Budapest (Chan et al, 2002), dos son las estrategias seguidas para la implantación y el desarrollo del Movimiento Open Access: la publicación de revistas en Open Access o el traspaso del modelo antiguo a la edición en abierto; y, por otro lado, "la creación de los denominados hasta ahora "Repositorios Institucionales (IR) en Open Access. Ambas estrategias de trabajo son denominadas ahora como "Ruta oro" y "Ruta verde" respectivamente" (Sánchez y Melero, 2007, p. 2).

La denominación de repositorio institucional si bien al principio tenía connotaciones de ser sólo para instituciones de investigación o universitarias se ha ampliado, estableciendo una definición mucho más amplia como señala (MacColl, Jones y Andrew, 2006, p. 1): "Los repositorios institucionales han surgido de las universidades, pero también se están extendiendo a otros tipos de organizaciones educativas, como los colegios e institutos de investigación".

Así, los repositorios institucionales han derivado en repositorios temáticos en donde se recopila documentos digitales de investigación de una institución o de una temática.

En la idea inicial de creación del repositorio RIARTE surgieron varias justificaciones del por qué realizarlo y aparecen en la génesis de éste (Consejo General de la Arquitectura Técnica de España, 2018):

- Unidad de todos los documentos almacenados que actualmente se encuentran dispersos en distintas bases de datos, repositorios o bibliotecas, lo cual permite una fácil recuperación.

- Recuperación de aquellos documentos que, o bien porque sus registros han desaparecido o nunca han sido registrados en bases en red, no estaban visibles.

- Preservación a largo plazo de los registros y/o los documentos.

- Visibilidad universal de los trabajos.

- Una comunicación a la sociedad en general y al mundo profesional y docente de la producción científica realizada por los AT.

- Una identificación del corpus documental de la profesión, con verificación de las áreas de mayor interés, evolución y perspectivas.

- Un registro que permite el análisis y futuras investigaciones desde diversos ámbitos de nuestra producción científico-técnica.

- Un interés mucho más creciente de toda la comunidad científica sobre nuestra profesión y la investigación generada.

- Una interoperatibilidad entre distintas bases de datos que facilita la recuperación de contenidos desde distintos repositorios y la incorporación a redes nacionales e internacionales de repositorios. 
- Una evaluación de los contenidos registrados respecto a su uso por los usuarios del repositorio. Esto también produce una mayor difusión del trabajo de los autores.

- El fortalecimiento del prestigio y reconocimiento de aquellos trabajos de calidad contrastada y con gran interés por los usuarios, anexo a los autores.

- Una convergencia de esfuerzos desde el Consejo y los Colegios, con la Universidad, e intercambio de criterios e iniciativas de proyectos futuros.

Hay también seis funciones consecuencia de la anteriores:

- la recuperación y puesta en valor de aquellos documentos que, por la escasa difusión del soporte o por su no registro, tenían alta obsolescencia.

- La difusión a través de redes nacionales e internaciones de portales y bases de datos de los documentos depositados en RIARTE.

- El tener una base de estudio de las áreas de investigación de la AT y, por ende, de la construcción en general, transversalmente en el estado de la cuestión y longitudinalmente a lo largo del tiempo.

- El reconocimiento a los investigadores a través de los índices de impacto inherentes al repositorio en donde su usabilidad a través de los accesos queda visible.

- El poder usar para la docencia una base de datos con información contrastada y actual de múltiples temas que, en la mayoría de los casos están dispersos.

- El aunar el mundo profesional, (revistas y congresos colegiales) con el investigador (investigadores docentes) con las connotaciones positivas que tiene la unificación del corpus documental.

El repositorio RIARTE es parte esencial también de la producción científica de la AT que se va incorporando año tras año, con la información de la base de datos que se realiza para el Observatorio de la AT (Durán, 2020), complementándose uno con otro.

Es una iniciativa única en España en donde cada día crece la cantidad y calidad de los documentos aportados. Y crece el uso de su base de datos como fuente de información de primer orden del mundo de investigación y profesional alrededor del sector de la construcción.

Además, y dada su interoperatibilidad y calidad, ya ha sido aceptada en redes nacionales e internacionales. Así, cualquier documento registrado en RIARTE, pasa a esas redes:

- $\quad$ RECOLECTA (FECYT. Ministerio de Ciencia e Innovación, 2021):

Recolecta, o Recolector de Ciencia Abierta, es el agregador nacional de repositorios de acceso abierto. En esta plataforma se agrupan a todas las infraestructuras digitales españolas en las que se publican y/o depositan resultados de investigación en acceso abierto.

Recolecta nació en el año 2007 fruto de la colaboración entre la Fundación Española para la Ciencia y la Tecnología (FECYT) y la Red de Bibliotecas Universitarias (REBIUN) de la CRUE. 
- HISPANA (Gobierno de España. Ministerio de Cultura y Deporte, 2021): Hispana es el portal de acceso al patrimonio digital y el agregador nacional de contenidos a Europeana.

Reúne las colecciones digitales de archivos, bibliotecas y museos españoles.

- $\quad$ EUROPEANA (Comisión Europea. European Digital Library Network, 2021): Europeana (Biblioteca Digital Europea) fue lanzada por la Comisión Europea en noviembre de 2008 , con dos millones de volúmenes, iniciando un proyecto que pretende crear un portal de la cultura europea digitalizada y de fácil acceso para el usuario. En la actualidad tiene ya más de 50 millones de documentos.

- GOOGLE ACADÉmico (Google Inc., 2021): Google Académico (Google Scholar) es un buscador que permite localizar documentos de carácter académico como artículos, tesis, libros, patentes, documentos relativos a congresos y resúmenes. Se alimenta de información procedente de diversas fuentes: editoriales universitarias, asociaciones profesionales, repositorios de preprints, universidades y otras organizaciones académicas.

Por ello cualquier documento indizado en RIARTE pasa a esta disponible en cualquier ordenador del mundo con los servidores de búsquedas en estos portales.

Así, RIARTE se transforma, también, en una herramienta muy útil para la docencia. Los materiales digitales recopilados son elementos de contenido con acceso fácil e intuitivo por parte de los alumnos y recopilan información normalmente diseminada y sin visibilidad.

Por lo tanto (Gewerc et al, 2006) no se trata sólo de localizar los recursos existentes en sus lugares de depósito (repositorios), sino de entender que la utilización pertinente de éstos requiere de apoyos formativos potentes para ayudar al profesorado universitario a realizar el cambio de paradigma de enseñanza para el aprendizaje demandado. Estos apoyos formativos deben ir dirigidos a que los docentes adquieran herramientas metacognitivas para posibilitar su aprendizaje permanente y a considerar y proporcionar oportunidades para la investigación sobre su práctica docente.

\section{Objetivos}

El objetivo general es el análisis del repositorio RIARTE desde el punto de vista documental y de usabilidad.

Los específicos son:

1. Analizar el número de documentos totales en RIARTE y evolución.

2. Clasificar la tipología de documentos registrados.

3. Estudiar el acceso por parte de los usuarios a RIARTE.

4. Determinar los documentos con mayor acceso.

5. Identificar a los autores con más impacto 


\section{Metodología}

Se ha tomado como origen de datos los que recoge el repositorio RIARTE y los sucesivos informes que se han venido elaborando sobre su funcionamiento. El espacio temporal ha sido desde Abril 2018 a Junio 2021.

Se usarán variables bibliométricas cuantitativas respecto a número de documentos y cualitativas respecto a la tipología de documentos.

Y variables altmétricas con el número de visualizaciones registradas.

Respecto a los datos del repositorio se han registrado con la siguiente metodología:

- Las tesis doctorales contemplan el total de las registradas por AT desde el inicio de la carrera (año 1.978).

- Los artículos técnicos dimanan de los registros de los artículos de revistas colegiales desde el año 2010.

- Las comunicaciones técnicas de las realizas en los Congresos CONTART 20062009-2010-2016-2018-2020.

- Los artículos y comunicaciones científicas de la producción de investigación en los años 2018-2019 de la AT.

Según la técnica de recogida de datos es una investigación descriptiva, dado que se pretende dar una visión general sobre RIARTE y sus contenidos.

La orientación de la investigación es aplicada, para el uso del repositorio y como herramienta docente.

La finalidad es descriptiva, dado que se representa RIARTE el fin último de esta comunicación es mostrarlo como herramienta docente y de visualización de contenidos.

Las variables que se han usado son cuantitativas (número de documentos, autores, descriptores) y cualitativas (visualizaciones).

El marco temporal es todos los documentos registrados en RIARTE desde su inicio hasta Junio de 2021. La investigación es tanto sincrónica porque se analiza el repositorio en el estado actual como diacrónica, dado que se estudia su evolución desde la creación.

\section{Desarrollo de la innovación y Resultados}

\subsection{Documentos}

El número total de documentos registrados hasta Junio de 2021 es de 1.811. La evolución a lo largo del tiempo ha sido la siguiente: 


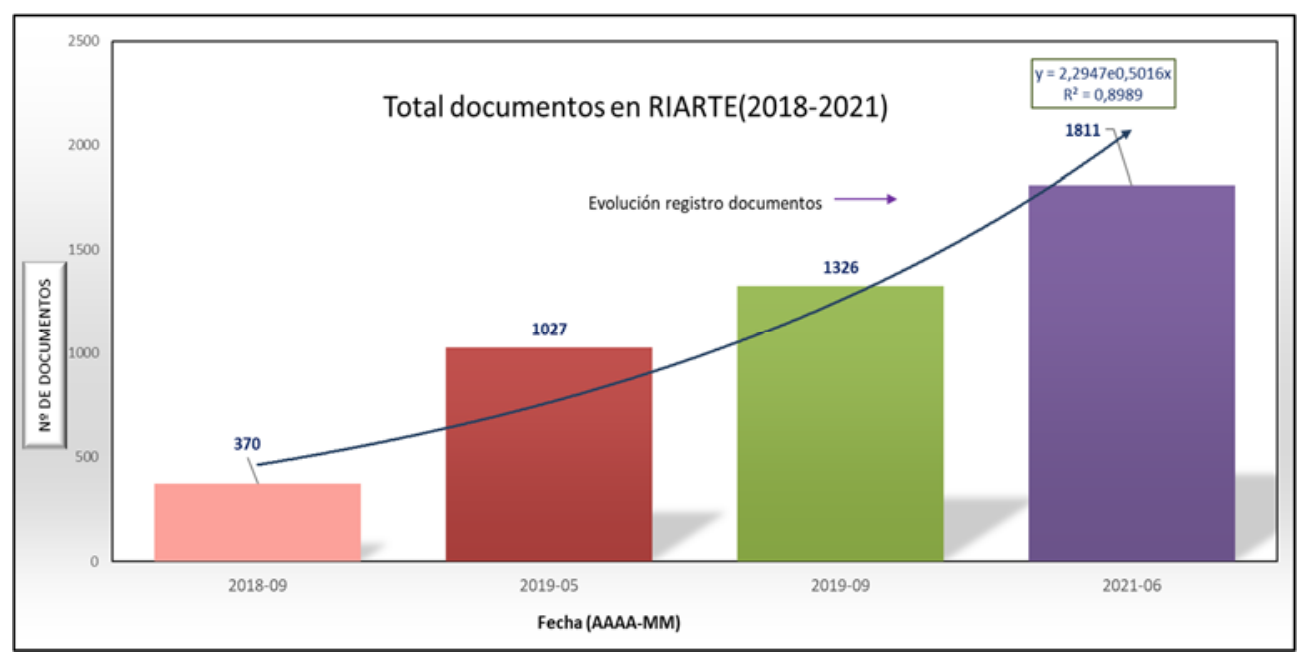

Figura 1. Total documentos en RIARTE (2018-2021). Origen: Elaboración propia

Como se observa, el ritmo de crecimiento de documentos es constante a lo largo del tiempo. En la actualidad se está registrando tanto la producción científica de 2020, como las actualizaciones de las revistas. Además, los autores cada vez más, piden la inclusión de toda su producción científica en RIARTE, dada su visibilidad y facilidad de acceso.

\subsection{Tipología de documentos}

El análisis actual de los documentos en RIARTE es:

Tabla 1. Total documentos en RIARTE por tipología. Origen: Elaboración propia

\begin{tabular}{lcc}
\multicolumn{1}{c}{ Tipos de documentos } & Totales & $\%$ \\
\hline TESIS DOCTORALES & 383 & $21,63 \%$ \\
\hline ARTÍCULOS CIENTÍFICOS & 192 & $10,84 \%$ \\
\hline ARTÍCULOS TÉCNICOS & 836 & $47,20 \%$ \\
\hline COMUNICACIONES CIENTÍFICAS & 45 & $2,54 \%$ \\
\hline COMUNICACIONES TÉCNICAS & 315 & $17,79 \%$ \\
\hline
\end{tabular}

Como se comprueba el porcentaje mayor es artículos técnicos. Son los registrados de las revistas colegiales dado que se han recogido todos los realizados desde el 2010 al 2021. Las tesis doctorales están en su totalidad y las comunicaciones técnicas, las existentes desde el año 2009 hasta el último CONTART 2020. 
Los artículos y comunicaciones científicas sólo se han registrado las del 2018-2019. Se está actualizando la base para incorporar las del 2020 y años anteriores.

Para el estudio del análisis temporal se han tomado los registros por tipología documental.

La evolución a lo largo del tiempo ha sido la siguiente:

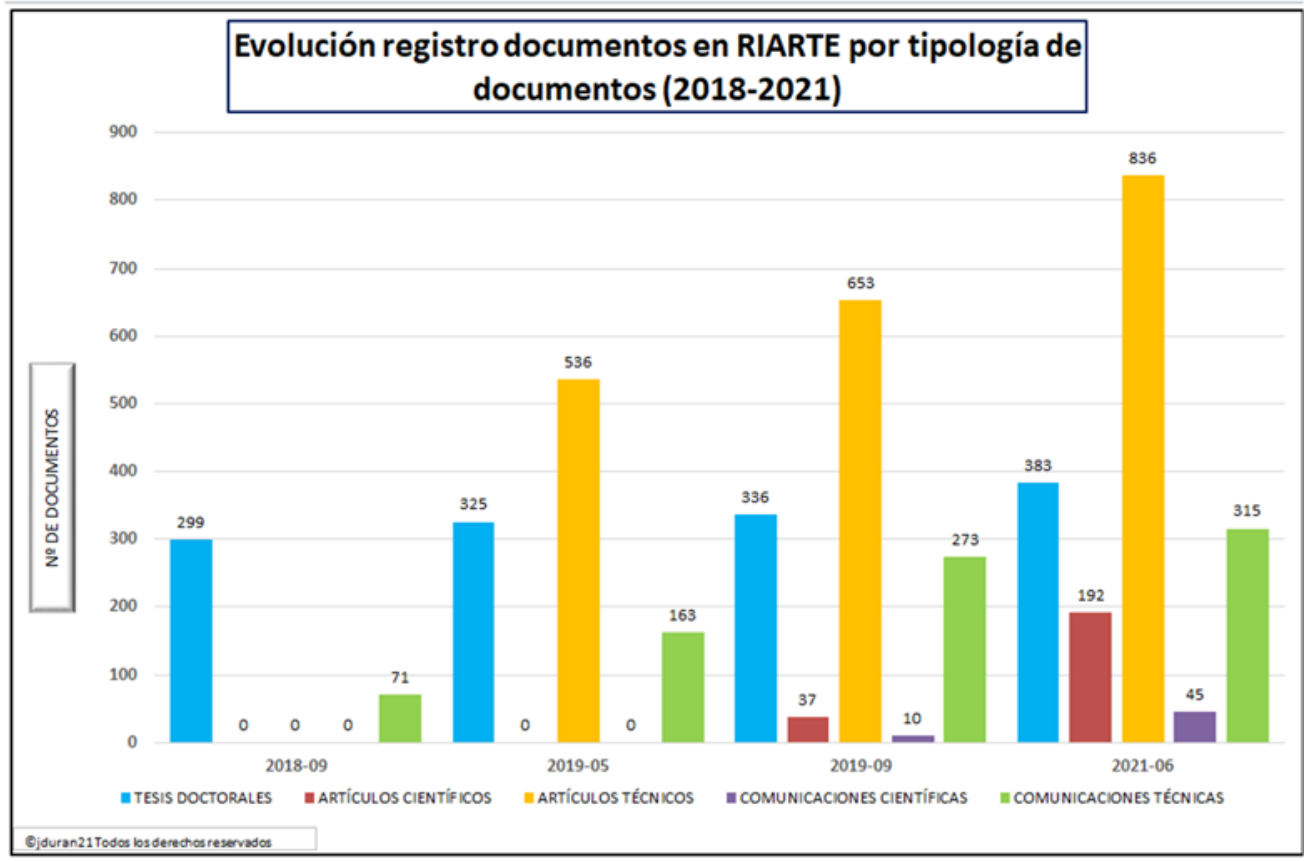

Figura 2. Evolución registro documentos en RIARTE por tipología documentos 2018-2021. Origen: Elaboración propia

\subsection{Autores}

El total de autores registrados ha sido de 426.

Las razones por tener más documentos pueden ser varias. Una es que en algunas revistas colegiales todos los artículos los firman autores de la misma revista y otra que algunos investigadores han pedido registrar toda su producción científica.

Tabla 2. Autores con más documentos registrados en RIARTE. Origen: Elaboración propia

\begin{tabular}{|lc|}
\hline \multicolumn{1}{|c}{ Autor } & $\begin{array}{c}\mathbf{N}^{\circ} \\
\text { Documentos }\end{array}$ \\
\hline Macías Bernal, Juan Manuel & 19 \\
Río Merino, Mercedes del & 15
\end{tabular}


RIARTE. El repositorio científico y profesional de la Arquitectura e Ingeniería. Contenidos y avances RIARTE. The scientific and professional repository of Architecture and Engineering. Contents and advances

\begin{tabular}{ll} 
Bienvenido Huertas, David & 13 \\
\hline Alejandre Sánchez, Francisco Javier & 11 \\
\hline Moyano Campos, Juan José & 11 \\
\hline Prieto Ibáñez, Andrés José & 10 \\
\hline Vidales Barriguete, Alejandra & 9 \\
\hline Piña Ramírez, Carolina & 9 \\
\hline Rodríguez Sáiz, Ángel & 8 \\
\hline Silva, Ana Catarina Ferrereira da & 8 \\
\hline González García, María de las Nieves & 6 \\
\hline
\end{tabular}

\subsection{Impacto}

Como impacto ha medido el número de visualizaciones de los documentos. El ranking creado es el siguiente:

Tabla 3. Documentos con más visualizaciones en RIARTE. Origen: Elaboración propia

\section{Título del documento}

Identificación de hogares vulnerables a partir del concepto pobreza energética. Indicador y modelo de evaluación
Visualizaciones

4.322

1.094

959

930

Lean Construction y la planificación colaborativa. Metodología del Last

Planner® System

El rol de las buenas prácticas educativas en los centros docentes :

821

organización, funcionamiento y docencia

Nueva metodología de evaluación de riesgos laborales adaptada a obras

800

de edificación: Nivel de la acción preventiva

Análisis cualitativo y cuantitativo de los fallos de diseño y ejecución en los

768

proyectos de cubiertas y fachadas y de su incidencia patológica en los

edificios de España

Intervención de los Arquitectos Técnicos en cálculos de estructuras

709

Sistema de gestión de residuos de construcción y demolición en obras de

682

edificación residencial. Buenas prácticas en la ejecución de obra

Aplicación del $8 \mathrm{~d}$ y de los principios Lean para la mejora de la seguridad y

674

prevención de obras de edificación 


\subsection{Descriptores}

En este repositorio el valor fundamental, aparte de lo mencionado es el registro de cada documento con descriptores libres y descriptores de un tesauro normalizado de la UNESCO, SKOS de Ciencia y Tecnología.

Con ello se parte de una base muy importante para análisis de redes, temas preferentes, clúster más relacionados, etc.

El total de descriptores libres que ahora mismo se ha registrado es de 3.192 y su análisis se presta a múltiples estudios.

Por ejemplo, respecto a tipologías de obras se han registrado los siguientes datos:

Tabla 4. Tipos de obra registrados en RIARTE. Origen: Elaboración propia

\begin{tabular}{|cc|}
\hline Descriptor tipo de obra & Documentos \\
\hline Obras de rehabilitación & 174 \\
\hline Obra nueva & 118 \\
\hline Obras de restauración & 68 \\
\hline Obras de reforma & 20 \\
\hline Obras de ampliación & 17 \\
\hline Obras de reparación & 17 \\
\hline Obras de consolidación & 9 \\
\hline Obras de reconstrucción & 4 \\
\hline
\end{tabular}

Como se comprueba, los documentos en su mayor parte están centrados en rehabilitación, después en obra nueva y finalmente en restauración. Es sintomático que la tendencia es la misma en el sector de la construcción según distintos informes (CESCE, 2020) y la rehabilitación aumenta su porcentaje años tras año, frente a la obra nueva en edificación.

\subsection{Factor de impacto}

Se han recogido los datos sobre el uso de RIARTE, por parte de los usuarios. Partíamos de 20.000 visualizaciones en el año 2019 , después de un año de funcionamiento.

A día de hoy, 30 de junio de 2021, los datos son: 
- Visitas a ítems (visualizaciones humanos):

- Visitas robots (tipo Google o buscadores portales): 2.140 .031

- Descargas de documentos:

El crecimiento ha sido exponencial y denota el interés del sector de la construcción en esta base de datos y que es una herramienta formidable para la transferencia de conocimiento desde la Arquitectura Técnica a la sociedad en general.

\section{Conclusiones}

Las conclusiones más importantes del presente trabajo son las siguientes:

1. El número de documentos en RIARTE en tres años ha aumentado de forma progresiva incorporando documentos técnicos y científicos.

2. Los documentos más registrados son artículos técnicos, tesis doctorales y comunicaciones técnicas.

3. El número de visualizaciones se ha multiplicado pasando el primer año de 8.000 a las casi 300.000 de la actualidad. Y las descargas también han sido muy importantes (95.631), lo que denota la usabilidad y visibilidad para los autores y usuarios.

4. Los documentos con mayor número de visualizaciones están relacionados con los temas más emergentes en la construcción: pobreza energética, bioconstrucción, programación y seguimiento de control de calidad, Lean Construction, Prevención de riesgos, patologías, cálculo de estructuras y $8 \mathrm{~d}$.

5. Los autores con mayor registro en RIARTE son Juan Manuel Macías Bernal, Mercedes del Río Merino y David Bienvenido Huertas. Los tres con gran producción científica y que, a su vez, publican tanto en revistas colegiales como en CONTART.

6. Respecto a los descriptores, éstos pueden reflejar la realidad de la Construcción en cuanto a distintos parámetros, como por ejemplo la tipología de obras que se realizan.

\section{Referencias}

AZORÍN, C., BERNAL, I., GOMEZ CASTAÑO, J., GUZMAN PEREZ, C., LOSADA YAÑEZ, M., MARIN DEL CAMPO, R., MARTINEZ GALINDO, F., MARTÍNEZ POUSA, C., MORILLO MORENO, J. C. y PRATS PRAT, J. (2021). Guía para la evaluación de repositorios institucionales de investigación. Fundación Española para la Ciencia y la Tecnología, FECYT.

BJÖRK, B., WELLINLAAKSO, M., WELLING, P., y PAEATAU, P. (2014). Anatomy of Green Open Access. Journal of the American Society for Information Science and Technology, vol. 65, issue 2, p. 237-250. doi:10.1002/asi.22963 
CESCE (2020). Construcción. Informe sectorial de la economía española. Madrid : Unidad de riesgos de CESCE.

CHAN, L., CUPLINSKAS, D., EISEN, M., FRIEND, F., GENOVA, Y., GUEDON, J.-C. y VELTEROP, J. (2002). The Budapest Open Access Initiative. Budapest.

COMISION EUROPEA. EUROPEAN DIGITAL LIBRARY NETWORK. (2021). EUROPEANA. $<$ https://www.europeana.eu/es > [Consulta: 28 de junio de 2021]

CONSEJO GENERAL DE LA ARQUITECTURA TECNICA DE ESPAÑA. (2018). Repositorio de investigación RIARTE. <http://www.riarte.es/page/about> [Consulta: 28 de junio de 2021]

DURAN ÁLVAREZ, J. (2020). Observatorio de la producción científica de la Arquitectura Técnica de España 2019. Granada: Unidad de Evaluación de la Actividad Científica. Vicerrectorado de Investigación y Transferencia. Universidad de Granada. doi:10.5281/zenodo.3902619

FECYT. MINISTERIO DE CIENCIA E INNOVACION. (2021). RECOLECTA. Recolector de ciencia abierta. https://recolecta.fecyt.es/portada?language=es [Consulta: 28 de junio de 2021]

FERRERAS FERNANDEZ, T., y MERLO VEGA, J. (2015). "Repositorios de acceso abierto: un nuevo modelo de comunicación científica. La Revista de la Sociedad ORL CLCR en el repositorio Gredos" en Revista de la Sociedad Otorrinolaringológica de Castilla y León, Cantabria y La Rioja, vol. 6, issue 12, p. 94-113.

GEWERC BARUJEL, A., PERNAS MORADO, E., RODRIGUEZ RODRIGUEZ, J., VIDAL PUGA, M., VILA SOBRINO, X., y AGRA PARDIÑAS, M. (2006). "La construcción de un repositorio de materiales abiertos reutilizables para apoyo a la docencia universitaria: MOREA" en Revista Lationamericana de tecnología educativa, vol. 5, issue 2, p.55-75.

GOBIERNO DE ESPAÑA. MINISTERIO DE CULTURA Y DEPORTE. (2021). HISPANA. Acceso en línea al patrimonio cultural. <https://hispana.mcu.es/es/inicio/inicio.do> [Consulta: 28 de junio de 2021]

GOOGLE INC. (2021). Google Académico. <https://scholar.google.es/schhp?hl=es> [Consulta: 28 de junio de 2021]

MACCOLL, J., JONES, R., y ANDREW, T. (2006). The institutional repository in the digital library. Chandos (Oxford) Publishing Ltd.

SANCHEZ, S., y MELERO, R. (2007). La denominación y el contenido de los Repositorios Institucionales en Acceso Abierto : base teórica para la "Ruta Verde". http://hdl.handle.net/10261/1487 